\title{
HEREDITARILY UNIVERSAL SETS
}

\section{ERIK ELLENTUCK*}

(Received 16 April 1973)

Communicated by J. N. Crossley

\begin{abstract}
An immune set is found such that the recursive equivalence type of its infinite subsets are universal in a very strong sense.
\end{abstract}

\section{Introduction}

Let $\omega$ be the non-negative integers and for $\xi \subseteq \omega$ let $\langle\xi\rangle$ be the recursive equivalence type of $\xi . \Lambda$ is of course the isols.

THEOREM 1. There is an immune $\eta \subseteq \omega$ such that for every infinite $\xi \subseteq \eta$ and $R \subseteq \omega \times \omega$ the graph of a function $r$, if $(\exists z \in \Lambda)(\langle\xi\rangle, z) \in R_{\Lambda}$ then $r$ is eventually recursive combinatorial.

THEOREM 2. There is an immune $\tau \subseteq \omega$ such that for every infinite $\xi \subseteq \tau$ and $R \subseteq \omega \times \omega$ the graph of a function $r$, if $(\exists z \in \Lambda)(\langle\xi\rangle, z) \in R_{\Lambda}$ then $r$ is eventually recursive increasing.

THEOREM 3. $\eta$ may be taken to be $\Delta_{2}^{1}$ and $\tau$ may be taken to be $\Pi_{1}^{0}$ (and retraceable).

Theorem 3 is a rather curious result. Theorems 1 and 2 look very much alike, the requirements on $\eta$ appearing only slightly stronger than those on $\tau$. We have no idea as to what degree the of $\eta$ might be, our $\tau$ on the other hand is of degree $0^{\prime}$. As an open problem we ask if better upper bounds or perhaps some lower bounds could be found for $\eta$ and $\tau$ ?

\section{Details}

Use lower case Greek letters for subsets of $\omega$ and let $\varnothing$ be the empty set. Define $(\alpha, \beta)^{\omega}=\{\alpha \cup \xi \mid \xi \subseteq \beta \wedge \xi$ is infinite $\},(\alpha, \beta)^{<\omega}=\{\alpha \cup \xi \mid \xi \subseteq \beta \wedge \xi$ is

\footnotetext{
* Supported by The Institute for Advanced Study
} 
finite $\}, \quad P=(\varnothing, \omega)^{\omega}, Q=(\varnothing, \omega)^{<\omega}$. A set $S \subseteq 2^{\omega}=P \cup Q$ is completely Ramsey if for every $\alpha \in Q$ and $\beta \in P$ there is a $\xi \in(\varnothing, \beta)^{\omega}$ such that $(\alpha, \xi)^{\omega} \subseteq S$ or $(\alpha, \xi)^{\omega} \subseteq 2^{\omega}-S$. The Galvin-Prikry theorem asserts that every Borel set is completely Ramsey (cf. Galvin and Prikry (1973)). We refer to this result as GP.

Let $R \subseteq \omega \times \omega$ be the graph of a function which is not eventually recursive combinatorial and let $F$ be a recursive $R$-frame. We use standard frame notation from Nerode (1961). If $\gamma \in F^{*}$ and $i<2$ let $C_{F}^{i}(\gamma)$ be the ith coordinate of $C_{F}(\gamma)$. dom and $r n g$ denote domain and range respectively. If $(\gamma, \varnothing) \in F^{*}$ put $\phi(\gamma)=$ $C_{F}^{0}(\gamma, \varnothing)$ and then define

$$
B(F)=\left\{\xi \in P \mid\left(\exists \gamma \in(\varnothing, \xi)^{<\omega}\right)\left(\forall \delta \in(\gamma, \xi)^{<\omega}\right) \phi(\delta) \subseteq \delta\right\} .
$$

Note that we always have $\delta \subseteq \phi(\delta)$ provided $\delta \in \operatorname{dom}(\phi)$. Let $\alpha \in Q$ and $\beta \in P$. Since $B(F)$ is clearly Borel, GP gives us an $\eta \in(\varnothing, \beta)^{\omega}$ such that $(\alpha, \eta)^{\omega} \subseteq B(F)$ or $(\alpha, \eta)^{\omega} \subseteq 2^{\omega}-B(F)$. That the latter always holds is given by

LEMMA 1. $(\alpha, \eta)^{\omega} \subseteq 2^{\omega}-B(F)$.

Proof. Assume $(\alpha, \eta)^{\omega} \subseteq B(F)$. We strive for a contradiction. Now $\alpha \cup \eta \in B(F)$ and hence there is a $\gamma \in(\varnothing, \alpha \cup \eta)^{<\omega}$ such that $\phi(\delta)=\delta$ for all $\delta \in(\gamma, \alpha \cup \eta)^{<\omega}$. Without loss of generality we may assume that $\alpha \subseteq \gamma$ so that $\phi(\delta)=\delta$ for all $\delta \in(\gamma, \eta)^{<\omega}$. By shrinking $\eta$ slightly we may also assume that $\gamma \cap \eta=\varnothing$. For the moment let $\delta$ range over $(\gamma, \eta)^{<\omega}$ and define $\psi(\delta)=C_{F}^{1}(\delta, \varnothing)$. Then $(\delta, \psi(\delta)) \in F$. Let $|\delta|$ be the cardinality of $\delta$. Since $R$ is single valued $|\delta|=\left|\delta^{\prime}\right|$ implies $|\psi(\delta)|=\left|\psi\left(\delta^{\prime}\right)\right|$. Also

$$
\left(\delta \cap \delta^{\prime}, \varnothing\right) \leqq(\delta, \psi(\delta)) \wedge\left(\delta^{\prime}, \psi\left(\delta^{\prime}\right)\right)=\left(\delta \cap \delta^{\prime}, \psi(\delta) \cap \psi\left(\delta^{\prime}\right)\right) \in F
$$

and thus $\psi\left(\delta \cap \delta^{\prime}\right) \subseteq \psi(\delta) \cap \psi\left(\delta^{\prime}\right)$. Since $\left(\delta \cap \delta^{\prime}, \psi\left(\delta \cap \delta^{\prime}\right)\right) \in F$ as well we have $\psi\left(\delta \cap \delta^{\prime}\right)=\psi(\delta) \cap \psi\left(\delta^{\prime}\right)$. Let $p$ be a one-one function mapping $\omega$ onto $\eta$. Define $\theta$ on $Q$ by $\theta(\lambda)=\psi(\gamma \cup p(\lambda))$. Then $|\lambda|=\left|\lambda^{\prime}\right|$ implies $|\theta(\lambda)|=\left|\theta\left(\lambda^{\prime}\right)\right|$ and $\theta\left(\lambda \cap \lambda^{\prime}\right)=\theta(\lambda) \cap \theta\left(\lambda^{\prime}\right)$. These properties are inherited from the corresponding ones for $\psi \cdot \theta$ is therefore a combinatorial operator inducing a combinatorial function $r: \omega \rightarrow \omega$ such that $(x+|\gamma|, r(x)) \in R$ for $x \in \omega$. Thus $R$ is the graph of an eventually combinatorial function. Let $B=\{(\lambda, \mu) \in Q \times Q \mid \lambda \cap \gamma$ $=\varnothing \wedge(\gamma \cup \lambda, \mu) \in F\}$ and $S=\{(x, y) \in \omega \times \omega|(\exists(\lambda, \mu) \in B) x=| \lambda|\wedge y=| \mu \mid\}$. $B$ and hence $S$ are r.e., the latter being the graph of $r$. Thus $R$ is the graph of an eventually recursive combinatorial function. Since $R$ was initially specified as not being such a relation, we have the desired contradiction.

Let $R \subseteq \omega \times \omega$ be the graph of a function and let $F$ be a recursive $R$-frame. $\phi$ is as above and define

$$
D(F)=\left\{\xi \in P \mid\left(\forall \gamma \in(\varnothing, \xi)^{<\omega}\right) \phi(\gamma) \subseteq \xi\right\} .
$$

Let $\alpha \in Q$ and $\beta \in P$. Since $D(F)$ is clearly Borel, GP gives us an $\eta \in(\varnothing, \beta)^{\omega}$ such 
that $(\alpha, \eta)^{\omega} \subseteq D(F)$ or $(\alpha, \eta)^{\omega} \subseteq 2^{\omega}-D(F)$. We relate $D(F)$ to the previous lemma by

LEMMA 2. If $(\alpha, \eta)^{\omega} \subseteq 2^{\omega}-B(F)$ then $(\alpha, \eta)^{\omega} \ddagger D(F)$.

Proof. Assume $(\alpha, \eta)^{\omega} \subseteq 2^{\omega}-B(F), \xi \in(\alpha, \eta)^{\omega}$ and $(\alpha \eta,)^{\omega} \subseteq D(F)$. Since $\xi \epsilon$ $2^{\omega}-B(F)$ there is a $\delta \in(\alpha, \xi)^{<\omega}$ such that $\delta \notin \operatorname{dom}(\phi)$ or $\phi(\delta) \notin \delta$. In the former case $\xi \notin D(F)$ and in the latter $\delta \cup(\xi-\phi(\delta)) \in(\alpha, \eta)^{\omega}-D(F)$, both of which contradict $(\alpha, \eta)^{\omega} \subseteq D(F)$.

Let $E(F)=\{\xi \in P \mid(\exists \zeta)(\xi, \zeta)$ is attainable from $F\}$.

LEMMA 3. $2^{\omega}-D(F) \subseteq 2^{\omega}-E(F)$.

Proor. An immediate consequence of definitions.

Let $S_{n} \subseteq 2^{\omega}$ be a sequence such that for each $n \in \omega, \alpha \in Q$ and $\beta \in P$ there is an $\eta \in(\varnothing, \beta)^{\omega}$ such that $(\alpha, \eta)^{\omega} \subseteq 2^{\omega}-S_{n}$. That we can find a uniform $\eta$ is given by

LEMMA 4. For each $\alpha \in Q$ and $\beta \in P$ there is an $\eta \in(\varnothing, \beta)^{\omega}$ such that $(\alpha, \eta)^{\omega} \subseteq 2^{\omega}-S_{n}$ for every $n \in \omega$.

Proof. Shrink $\beta$ slightly so that every element of $\alpha$ is less than every element of $\beta$. Let $\alpha_{0}=\alpha$ and choose $\eta_{0} \in(\varnothing, \beta)^{\omega}$ so that $\left(\alpha_{0}, \eta_{0}\right)^{\omega} \subseteq 2^{\omega}-S_{0}$. Now suppose we have defined $\alpha_{n}$ and $\eta_{n}$ such that every element of $\alpha_{n}$ is less than every element of $\eta_{n}$. Let $a_{n}$ be the least element of $\eta_{n}$. Set $\alpha_{n+1}=\alpha_{n} \cup\left\{a_{n}\right\}$ and choose $\eta_{n+1} \in\left(\varnothing, \eta_{n}-\left\{a_{n}\right\}\right)^{\omega}$ so that for each $\alpha_{0} \subseteq \gamma \subseteq \alpha_{n+1}$ we have $\left(\gamma, \eta_{n+1}\right)^{\omega} \subseteq$ $2^{\omega}-S_{n+1}$. Then $\eta=\cup \alpha_{n}$ has the required property.

ProOF OF THEOREM 1. Let $F_{n}$ be an enumeration of all recursive $R$-frames where $R \subseteq \omega \times \omega$ is the graph of a which is not eventually recursive combinatorial. Start with an immune set $\beta$ and use GP and lemma 1 to get an $\eta \in(\varnothing, \beta)^{\omega}$ such that $(\varnothing, \eta)^{\omega} \subseteq 2^{\omega}-B\left(F_{n}\right)$ and either $(\varnothing, \eta)^{\omega} \subseteq D\left(F_{n}\right)$ or $(\varnothing, \eta)^{\omega} \subseteq 2^{\omega} D\left(F_{n}\right)$. By lemma $2,(\varnothing, \eta)^{\omega} \subseteq 2^{\omega}-D\left(F_{n}\right)$ and by lemma $3,(\varnothing, \eta)^{\omega} \subseteq 2^{\omega}-E\left(F_{n}\right)$. Lemma 4 gives an $\eta$ which uniformly works for all $n \in \omega$. Thus if $R$ is as above and $\xi \in(\varnothing, \eta)^{\omega}$ then for no recursive $R$-frame $F$ and $\zeta[$ an $(\xi, \zeta)$ be attainable from $F$. This is the contrapositive of our theorem.

Let $j$ be the usual pairing function with $k, l$ as its first, second inverse. Order the elements of $Q$ according to their canonical indices so that we can effectively speak of a first, second... element of $Q$. Let $q_{n}(\alpha)$ be a partial recursive function of $n \in \omega$ and $\alpha \in Q$ which with index $n$ enumerates partial recursive functions mapping subsets of $Q$ into $\omega$. Put $q_{n}^{s}(\alpha)=y$ if $q_{n}(\alpha)=y$ in $s$ or fewer computation stages, otherwise we say that $q_{n}^{s}(\alpha)$ is undefined. Denote the largest element in $\alpha \in Q$ by $\max (\alpha)$. A retraceable function, $t$, is called hereditarily 1-meager if for every $e \in \omega$ there is an $m \in \omega$ such that for all $n>m$ and $\alpha \subseteq\{t(i) \mid i<n\}$ $q_{e}(\alpha)$ is undefined or $q_{e}(\alpha)<t(n)$. The following lemma is closely related to our 
proof (cf. Ellentuck (1973)) of McLaughlin's theorem on the existence of hereditarily retraceable isols (cf. McLaughlin (1967)).

LEMMA 5. There exists a hereditarily 1-meager function with cosimple range.

Proof. Our proof is a stage by stage construction of functions $t^{s}(n)$ whose limit $t(n)=\lim _{s} t^{s}(n)$ is hereditarily 1 -meager.

Stage $s=0$ : Let $t^{0}(0)=1$ and then go on to stage 1 .

Stage $s+1$ : As inductive hypothesis assume at the end of stage $s$ that we have defined $t^{s}(n)$ for $n \leqq s$, that $t^{s}(0)=1$, and that $k t^{s}(n+1)=t^{s}(n)$ for $n<s$. Search for the least $n \leqq s$, and for it the least $m<n$, and for them the least $\alpha \subseteq\left\{t^{s}(i) \mid i<n\right\}$ such that

$$
q_{m}^{s}(\alpha) \text { is defined and } t^{s}(n) \leqq q_{m}^{s}(\alpha) \text {. }
$$

If there is no such $(n, m, \alpha)$ go to case A below, otherwise go to case B.

CASE A. Let $t^{s+1}(x)=t^{s}(x)$ for $x \leqq s, t^{s+1}(s+1)=j\left(t^{s}(s), 0\right)$.

CASE B. Find the least $y$ such that

$$
\max \left\{q_{m}^{s}(\alpha), t^{s}(s)\right\}<j\left(t^{s}(n-1), y\right)
$$

(note that $n>0$ ) and let $t^{s+1}(x)=t^{s}(x)$ for $x<n, t^{s+1}(n)=j\left(t^{s}(n-1), y\right.$ ), and $t^{s+1}(x+1)=j\left(t^{s+1}(x), 0\right)$ for $n \leqq x \leqq s$. This completes stage $n+1$ of the construction. Now go on to stage $s+2$. It is easy to see that our inductive hypothesis is maintained as we pass through stages. $t(n)=\lim _{s} t^{s}(n)$ exists for every $n$ because $t^{s}(0)=1$ for every $s$, and once $t^{s}(n-1)$ has reached its final value $t^{s}(n)$ changes its value at most $n \cdot 2^{n}$ times. $t(0)=1$ and $k t(n+1)=t(n)$ by our inductive hypothesis and $t$ is one-one since $t(0) \neq 0$. Thus $t$ is retraceable, and the construction in case $B$ insures that $x \notin \operatorname{rng}(t)$ if and only if $(\exists x>s) x \notin \operatorname{rng}\left(t^{s}\right)$. This makes $\operatorname{rng}(t)$ co-r.e. The immunity of $\operatorname{mg}(t)$ follows from the meagerness of $t$. We demonstrate the latter. Let $m<n$ and choose a stage $r$ so large that $t^{r}(i)$ for $i \leqq n$ have reached their final values. There can be no $\alpha \subseteq\{t(i) \mid i<n\}$ such that $t(n) \leqq q_{m}(\alpha)$, otherwise $t^{r}(n)$ would subsequently change its value.

Proof of TheOREM 2. Let $\zeta=\operatorname{rng}(t), \sigma \in(\varnothing, \tau)^{\omega}$ and $s_{n}$ a strictly increasing enumeration of $\sigma$. Let $R \subseteq \omega \times \omega$ be the graph of a function $r$ for which $(\exists z \in \Lambda)(\langle\sigma\rangle, z) \in R_{\Lambda}$. Then there is an isolated $\zeta$ and a recursive $R$-frame $F$ such that $(\sigma, \zeta)$ is attainable from $F$. If $(\alpha, \varnothing) \in F^{*}$ put $\phi(\alpha)=\max C_{F}^{0}(\alpha, \varnothing)$ and let $A=\left\{\alpha \in Q \mid C_{F}^{0}(\alpha, \varnothing)=\alpha\right\}$. By applying Lemma 5 to $\phi$ we see that there is an $m \in \omega$ such that $\left\{s_{i} \mid i<n\right\} \in A$ for any $n>m$. Let $\psi(\alpha)=C_{F}^{1}(\alpha, \varnothing)$ for $\alpha \in A$. $A$ is a r.e. family of finite sets, $\psi$ is a partial recursive function taking finite sets into finite sets and $(\alpha, \psi(\alpha)) \in F$ for every $\alpha \in A$. If $\alpha, \alpha^{\prime} \in A$ and $\alpha \subseteq \alpha^{\prime}$ then $(\alpha, \varnothing) \leqq\left(\alpha^{\prime}, \psi\left(\alpha^{\prime}\right)\right.$ and hence $\psi(\alpha) \subseteq \psi\left(\alpha^{\prime}\right)$. Let $S=\{(a, b) \mid(\exists \alpha \in A) a=$ 
$|\alpha| \wedge b=|\psi(\alpha)|\} . S$ is r.e. subset of $R$ and the graph of a partial function whose domain contains all $n>m$. It is also the graph of an eventually increasing function by the monotonicity of $\psi$. Thus $r$ is eventually recursive increasing.

Proof of Theorem 3. We have already dealt with $\tau$. For $\eta$ notice that $(\varnothing, \eta)^{\infty} \subseteq 2^{\infty}-B(F)$ is a $\Pi_{1}^{1}$ predicate. Since ' $R$ is the graph of a function which is not eventually recursive combinatorial' is an arithmetical predicate, and there is an arithmetical enumeration of all recursive frames, we see that the condition required of $\eta$ in the proof of Theorem 1 is $\Pi_{1}^{1}$. By Addison's modification of the Kondo theorem (cf. Rogers (1967)) $\eta$ may be chosen as $\Delta_{2}^{\mathbf{1}}$.

We had originally hoped to get $\eta$ recursive in the ordinal notations. We have not been able to do so; however, such an attempt seems promising.

\section{References}

F. Galvin and K. Prikry 1973), 'Borel sets and Ramsey's theorem', J. Symbolic Logic 38, 193-198.

E. Ellentuck (1973), 'On the degrees of universal regressive isols,' Math. Scand. 32, 145-164.

T. McLaughlin (1967), 'Hereditarily regtaceable isols', Bull. Amer. Math. Soc. 73, 113-115.

A. Nerode (1961), 'Extensions to isols', Ann. of Math. 73, 362-403.

H. Rogers Jr. (1967), Theory of Recursive Finctions and Effective Computability, (McGraw-Hill, New York, 1967).

Rutgers, The State University

New Brunswick, New Jersey

U. S. A. 